Bull. Austral. Math. Soc.

Vol. 45 (1992) [91-103]

\title{
RINGS WHOSE ADDITIVE ENDOMORPHISMS ARE RING ENDOMORPHISMS
}

\author{
Manfred Dugas, Jutta Hausen and Johnny A. Johnson
}

\begin{abstract}
A ring $R$ is said to be an $A E$-ring if every endomorphism of its additive group $R^{+}$ is a ring endomorphism. Clearly, the zero ring on any abelian group is an $A E$-ring. In a recent article, Birkenmeier and Heatherly characterised the so-called standard $A E$-rings, that is, the non-trivial $A E$-rings whose maximal 2-subgroup is a direct summand. The present article demonstrates the existence of non-standard $A E$ rings. Four questions posed by Birkenmeier and Heatherly are answered in the negative.
\end{abstract}

\section{INTRODUCTION}

In 1977, Sullivan posed the problem of characterising all rings $R$ with the property that every endomorphism of its additive group $R^{+}$is, in fact, a ring homomorphism [9]. It is convenient to call such a ring an $A E$-ring [3]. In 1981, Kim and Roush characterised all finite $A E$-rings [7], and in a recent paper Feigelstock extended this characterisation to the $A E$-rings $R$ whose additive group is a torsion group [3]. Birkenmeier and Heatherly solved Sullivan's problem for the case that the 2-component $R_{2}$ of $R^{+}$is a direct summand [1]. Without explicitly addressing the problem, they hinted that this need not always be the case [1, Theorem $8(i i)]$, and posed four questions:

QUESTION I. Are all $A E$-rings commutative?

QUESTION II. Is every subdirectly irreducible homomorphic image of an $A E$-ring also an $A E$-ring?

QUESTION III. Is every homomorphic image of an $A E$-ring an $A E$-ring?

QUESTION IV. If $R$ is an $A E$-ring in which $x^{2}=0$ for each $x \in R$, is $R^{2}=0$ ?

We will show that the answer to each of these questions is negative. For this, we need to consider $A E$-rings $R$ whose 2-component is not a direct summand (we will term such $A E$-rings non-standard). It is shown that any non-standard $A E$-ring $R$ must be close to a zero ring in the sense that $R \cdot(t R+2 R)=0=(t R+2 R) \cdot R$ where $t R$ denotes the maximal torsion subgroup of $R^{+}$, and $R^{3}=0$. An example will

Received 31st January 1991

Copyright Clearance Centre, Inc. Serial-fee code: $0004-9729 / 92 \$ \$ A 2.00+0.00$. 
be constructed which demonstrates that non-standard $A E$-rings exist which are not zero rings. It should be noted that a similar construction yields an abelian group $G$ which supports $2^{2^{k_{0}}}$ pairwise non-isomorphic $A E$-rings. Thus, there is little hope for a complete solution to Sullivan's problem.

Abelian group notation will follow Fuchs' monographs [4, 5]. In particular, o(a) denotes the order of an element $a$ in the group $A$, and $|A|$ is the order of $A$; the subgroup of $A$ consisting of all elements of 2-power order is denoted by $A_{2}$, and $A[2]$ is the subgroup consisting of all elements of order at most 2 . As is customary, $2^{\omega} A=$ $\bigcap_{n<\omega} 2^{n} A$, and $R=I+J$ denotes the ring direct sum of the ideals $I$ and $J$. For ease of reference, we collect some results due to Feigelstock:

Lemma 1.1. [3] Let $R$ be an $A E$-ring. Then

(1) If $R^{+}=A \oplus B$ then $A$ and $B$ are ideals of $R$ and $R=A+B$.

(2) $R^{2} \subseteq R[2]$.

(3) If $R^{2} \neq 0$ then $R_{2}$ is reduced.

\section{Standard $A E$-Rings}

Given an abelian group $A$, a ring on $A$ is a ring $R$ such that $R^{+}=A$. The zero ring on $A$ is the ring $R$ on $A$ with trivial multiplication: $R^{2}=0$. Obviously, the zero ring on any abelian group $A$ is an $A E$-ring. By a non-trivial $A E$-ring we shall mean an $A E$-ring $R$ with $R^{2} \neq 0$.

Birkenmeier and Heatherly characterised the $A E$-rings $R$ whose 2-component is a direct summand [1, Theorem 4]. We have an alternate condition:

Proposition 2.1. Let $R$ be an $A E$-ring such that $R^{2} \neq 0$. Then $R_{2}$ is a direct summand of $R$ if and only if $R^{2} \nsubseteq 2^{\omega} R$.

Proof: By [1, Theorem 4], $R_{2}$ being a direct summand of $R$ implies $R_{2}$ bounded so that $2^{\omega} R=0$. Conversely, assume $R^{2} \nsubseteq 2^{\omega} R$. Frequent use will be made of 1.1. Let $x$ and $y$ be elements of $R$ such that $x y \notin 2^{\omega} R$. Then $x y \in R[2]$ has finite 2-height $n-1 \geqslant 0$. If $x y=2^{n-1} c$ for some $c \in R$ then $c$ has order $2^{n}$ and $[4, \mathrm{p} .117,27.1]$ implies $R^{+}=\langle c\rangle \oplus W$. Hence $R=\langle c\rangle \dot{+} W$. Let $p$ and $q$ be integers and $v, w \in W$ such that $x=p c+v$ and $y=q c+w$. Then $2^{n-1} c=x y=p q c^{2}+v w$ which implies $p q$ is odd and $c^{2}=2^{n-1} c$. Assume, by way of contradiction, that $R_{2}$ is unbounded. Since $R_{2}$ is reduced, there exists a decomposition $W=\langle d\rangle \oplus X$ with $o(d)=2^{m}$ for some $m \geqslant 2 n$. It follows that there is $f \in \operatorname{End}\left(R^{+}\right)$such that $f(d)=c$ and $f(c)=0$. Lemma 1(ii) of [1] implies $c^{2}=0$ which is a contradiction. By [1, Corollary 5], $R_{2}$ is a direct summand.

THEOREM 2.2. Let $R$ be a ring. Then $R$ is an $A E$-ring with $R^{2} \nsubseteq 2^{\omega} R$ if and 
only if

$$
R=\langle c\rangle+S+N
$$

with $o(c)=2^{n}, n$ a positive integer, $c^{2}=2^{n-1} c, S$ and $N$ zero rings with $2^{n-1} S=0$, $N_{2}=0$ and $N=2 N$.

Proof: Again, 1.1 will be used without mention. Assume, firstly, that $R$ is an $A E$-ring such that $R^{2} \nsubseteq 2^{\omega} R$. By 2.1 , we may apply Theorem 4 of [1]. Using the notation of [1], it remains to show that $N$ is 2-divisible. Since $N_{2}=0$, the torsion subgroup $t N$ of $N$ is 2-divisible. Assume $N \neq 2 N$. Then $N / t N$ is a torsion-free abelian group which is not 2-divisible and as such has a quotient group isomorphic to $Z\left(2^{n}\right) \simeq C$. Let $g \in H o m\left(N^{+}, R_{2}^{+}\right)$with $g(N)=C$. By [1, Theorem 4(iii)], $g(N) \cdot R_{2}=0$ contradicting $C \cdot C \neq 0$. Thus $N=2 N$. For the reverse implication, assume $R$ is as stated. Then $R_{2}$ is a direct summand, $R_{2}$ is bounded, and the 2divisibility of $N$ implies $\operatorname{Hom}\left(N^{+}, R_{2}^{+}\right)=0$. By [1, Theorem 4] $R$ is an $A E$-ring. $]$

We shall call the $A E$-rings described in 2.1 the standard $A E$-rings. Thus $R$ is standard if $R^{2} \nsubseteq 2^{\omega} R$. Every non-trivial torsion $A E$-ring is standard [3].

One verifies that an abelian group $A$ which supports one standard $A E$-ring will support no other $A E$-ring except for the zero ring. This will be different in the case of non-standard $A E$-rings.

\section{NON-STANDARD $A E$-RINGS}

An $A E$-ring $R$ is called non-standard if $R^{2} \subseteq 2^{\omega} R$ and $R$ is not trivial, that is, $R^{2} \neq 0$. We have the following result.

Proposition 3.1. Let $R$ be a non-standard $A E$-ring. Then necessarily $R$. $(t R+2 R)=0=(t R+2 R) \cdot R$. In particular, $R^{3}=0$.

Proof: Assume, by way of contradiction, there exist elements $x \in t R$ and $r \in R$ such that $R \cdot(x+2 r) \neq 0$. Since $2 R^{2}=0$ it follows that $R \cdot x \neq 0$ and $R \cdot x=R \cdot a$ for some $a \in R$ of 2-power order. Pick $c \in R_{2}$ of minimal order satisfying $R \cdot c \neq 0$. Let $o(c)=2^{n}$. Then $n$ is positive and, by 1.1(2), $c$ has height zero. We claim that $\langle c\rangle$ is a direct summand of $R^{+}$. By $[4$, p.117, 27.1], it suffices to show that $\langle c\rangle \cap 2^{n} R=0$. Assume there exists an integer $m$ and an element $s \in R$ such that $2^{m} c=2^{n} s \neq 0$. Then $1 \leqslant m<n$ which implies $c-2^{n-m} s \in R_{2}$ of order at most $2^{m}$. Since $R \cdot\left(c-2^{n-m} s\right)=R \cdot c \neq 0$, this contradicts the minimality of the order of $c$. Thus $\langle c\rangle$ is a direct summand of $R^{+}$. From 1.1(1) we have $0 \neq R \cdot c \subseteq\langle c\rangle$. Since (c) $\cap 2^{\omega} R=0$, this is a contradiction. Similarly, $(t R+2 R) \cdot R=0$.

In order to demonstrate the existence of non-standard $A E$-rings the following theorem is needed. Given two abelian groups $G$ and $H$, the set of all homomorphisms 
$\phi: G \rightarrow H$ with $I m \phi$ a bounded torsion group is denoted by $H \operatorname{om}_{b}(G, H)$. Throughout, $P$ denotes Prüfer's 2-group and $a \in P$ has the property that $\langle a\rangle=2^{\omega} P \simeq Z(2)[4$, p. 150]. The set of all endomorphisms of a group $G$ which are integral multiplications is denoted by $\mathrm{Z} \cdot 1_{G}$.

Theorem 3.2. Let $G$ be an abelian group such that $t G=P, G \neq t G+2 G$, and assume that $E n d G=\mathrm{Z} \cdot 1_{G} \oplus \operatorname{Hom}_{b}(G, P)$. Then $G$ supports a non-standard $A E$-ring. In fact:

(1) if $K$ is a subgroup of $G$ containing $t G+2 G$ and $X$ is a set of elements in $G$ which is minimal with respect to the set $\{x+K \mid x \in X\}$ being a basis of $G / K$, then, given any map $f: X \times X \rightarrow 2^{\omega} P$, there exists an $A E$-ring $R$ with $R^{+}=G$ such that $R \cdot K=0=K \cdot R$ and $x \cdot y=f(x, y)$ for all $(x, y) \in X \times X$;

(2) if $G /(t G+2 G)$ has infinite dimension $\delta$ over $\mathrm{Z}_{2}$, then there exist $2^{\delta}$ pairwise non-isomorphic $A E$-rings on $G$.

Proof: Let $\pi: G \rightarrow G / K$ denote the natural epimorphism, and consider any $\eta \in H o m\left(G / K \otimes G / K, 2^{\omega} P\right)$. Define $\mu: G \otimes G \rightarrow 2^{\omega} P$ by $\mu=\eta \circ(\pi \otimes \pi)$. Then $\mu$ is a homomorphism and, defining a multiplication ${ }_{\eta}$ on $G$ by $g \cdot{ }_{\eta} h=\mu(g \otimes h)$ for all $g, h \in G$ makes $G$ into a ring $R=R_{\eta}$ (which, in general, need not be associative) [5, p. 281]. In our case, $g \cdot{ }_{\eta} h=\eta[(g+K) \otimes(h+K)]$ which implies $R \cdot_{\eta} K=0=K \cdot_{\eta} R$; by construction, $R \cdot{ }_{\eta} R \subseteq I m \eta \leqslant 2^{\omega} P \leqslant K$ Hence, $R^{3}=0$ which implies that $R$, in fact, is associative. We claim that, for every $\eta \in \operatorname{Hom}\left(G / K \otimes G / K, 2^{\omega} P\right)$, the ring $R=R_{\eta}$ is an $A E$-ring. Let $\varepsilon$ be an endomorphism of $R^{+}=G$. By hypothesis, $\varepsilon=n \cdot 1_{G}+\beta$ with $n \in \mathbf{Z}$ and $\operatorname{Im} \beta$ bounded. It follows that $\beta\left(2^{\omega} P\right)=0$ and $\beta(G) \subseteq K$. Let $g, h \in G$. Then, skipping the subscript $\eta$, we have $\varepsilon(g) \cdot \varepsilon(h)=(n g+\beta(g)) \cdot(n h+\beta(h))=n^{2} g h$, and $\varepsilon(g h)=n g h+\beta(g h)=n g h$. Since $g h \in 2^{\omega} P=\langle a\rangle$ and $n a=n^{2} a$ for all integers $n$, we have shown that $R$ is an $A E$-ring. In order to verify (1), let $f: X \times X \rightarrow 2^{\omega} P$ be a map. Since the set $B=\{\pi(x) \otimes \pi(y) \mid(x, y) \in X \times X\}$ is a basis for the vector space $G / K \otimes G / K[5$, p.255, (I) and (H)] and $(x, y) \mapsto \pi(x) \otimes \pi(y)$ defines a bijection between $X \times X$ and $B$, there exists a homomorphism $\sigma: G / K \otimes G / K \rightarrow 2^{\omega} P \simeq Z(2)$ such that, for all $(x, y) \in X \times X, \sigma[\pi(x) \otimes \pi(y)]=f(x, y)$. If $S=R_{\sigma}$ then $S$ is an $A E$-ring on $G$ and, for all $x, y \in X$, we have $x \cdot \sigma y=\sigma[\pi(x) \otimes \pi(y)]=f(x, y)$ as claimed. In order to verify (2), note that

$$
\operatorname{Hom}\left(G / K \otimes G / K, 2^{\omega} P\right) \simeq \prod_{(x, y) \in X \times X} \operatorname{Hom}(\langle\pi(x)\rangle \otimes\langle\pi(y)\rangle,\langle a\rangle)
$$

which has cardinality $2^{\delta}$ if $|X|=\delta$ is infinite. Let $\eta, \sigma \in \operatorname{Hom}(G / K \otimes G / K,\langle a\rangle)$. Assume the resulting $A E$-rings $R_{\eta}$ and $R_{\sigma}$ are isomorphic and let $\alpha$ be a ring isomorphism between them. Then $\alpha$ would have to be an automorphism of the underlying 
additive group, $G$, in particular $\alpha=n \cdot 1_{G}+\beta$ with $\beta(G)$ bounded. It follows that $\beta(a)=0, \beta(G) \leqslant K$ and $a=\alpha(a)=n(a)$ so that $n$ is odd. Being a ring isomorphism, we have $\alpha\left(g \cdot_{\eta} h\right)=\alpha(g) \cdot_{\sigma} \alpha(h)$ for all $g, h \in G$ and consequently $\eta(\pi(g) \otimes \pi(h))=$ $n \eta(\pi(g) \otimes \pi(h))=n\left(g \cdot_{\eta} h\right)=\alpha\left(g \cdot_{\eta} h\right)=(n g+\beta(g)) \cdot_{\sigma}(n h+\beta(h))=(n g) \cdot_{\sigma}(n h)=$ $n^{2}\left(g \cdot_{\sigma} h\right)=(g \cdot \sigma h)=\sigma(\pi(g) \otimes \pi(h))$. It follows that $\eta=\sigma$. This completes the proof.

The last section of our paper is devoted to the proof of

Proposition 3.3. There exists an abelian group $G$ satisfying the hypothesis of 3.2 such that $G /(t G+2 G)$ has rank two.

Proposition 3.3 enables us to answer all four question posed in the Introduction in the negative:

Corollary 3.4. Not every $A E$-ring is commutative.

Corollary 3.5. There exists an $A E$-ring $R$ such that $r^{2}=0$ for all $r \in R$ but $R^{2} \neq 0$.

Proof of 3.4 AND 3.5: Let $G$ be the group of 3.3. Put $t G+2 G=K$ and let $x, y \in G$ such that $G / K=\langle x+K\rangle \oplus\langle y+K\rangle$. By 3.2, there exists an $A E$-ring $R$ with $R^{+}=G$ such that $x \cdot y=a$ and $y \cdot x=0$. Thus $R$ is not commutative, proving 3.4. Similarly, there exists and $A E$-ring $S$ with $S^{+}=G$ such that $x^{2}=0=y^{2}$ and $x \cdot y=a=y \cdot x$. Let $r \in R$. Then $r=m x+n y+k$ for some integers $m$ and $n$ and some $k \in K$, and

$$
r \cdot r=m^{2} x^{2}+m n x \cdot y+n m y \cdot x+n^{2} y^{2}=2 m n a=0
$$

proving 3.5.

Thus, Questions I and IV of [1] have negative answers. As pointed out by Birkenmeier and Heatherly, an affirmative answer to II would imply that every $A E$-ring is commutative. This follows from the fact that every subdirectly irreducible $A E$-ring is commutative [1, Theorem 13(iii)] and the well known theorem that every ring $R$ is a subdirect sum of a product of subdirectly irreducible quotient rings of itself $[8, p .129$, Theorem 34]. It thusly follows from 3.4 that Question II has a negative answer as well (and, hence, so does III).

In order to give a concrete example we include

PropostTION 3.6. Not every subdirectly irreducible homomorphic image of an $A E$-ring is an $A E$-ring.

Proof: Let $G$ be the group of 3.3. Then there exists $x \in G$ and a subgroup $A$ of $G$ such that $t G+2 G \leqslant A$ and $G / A=\langle x+A\rangle \simeq Z(2)$. By 3.2, there exists an $A E$-ring $R$ on $G$ with $x^{2}=a$ and $R \cdot A=0=A \cdot R$. Let $B$ be a basic subgroup of $P$ such 
that $P / B \simeq Z\left(2^{\infty}\right)$. Then $(P / B)[2]=\langle a+B\rangle$. Since $x \notin A$, the order of $x$ must be infinite so that $\langle x+B\rangle \cap P / B=0$. Divisible subgroups are absolute direct summands. Thus $G / B=P / B \oplus C / B$ for some subgroup $C$ of $G$ with $B \leqslant C$ and $x \in C$. Put $A \cap C=I$. Then $I$ is an ideal of $R$ and $x \notin I$ since $x \notin A$. Also, since $a \in P \backslash B$, we have $a \notin C$ so that $a \notin I$. By construction, $(x+I)^{2}=x^{2}+I=a+I \neq 0$ which shows that $R / I$ is not a zero ring. Since $G=A+\langle x\rangle=A+C$ we have

$$
A / I=A /(A \cap C) \simeq(A+C) / C=G / C \simeq(G / B) /(C / B) \simeq P / B \simeq Z\left(2^{\infty}\right)
$$

Thus $(R / I)^{+}=G / I=A / I+\langle x+I\rangle$ and $x \notin I$ but $2 x \in A \cap C=I$ which implies $(R / I)^{+}=A / I \oplus\langle x+I\rangle \simeq Z\left(2^{\infty}\right) \oplus Z(2)$. It follows from $1.1(3)$ that $R / I$ cannot be an $A E$-ring. In order to verify that $R / I$ is subdirectly irreducible, it suffices to show that every nonzero ideal of $R / I$ contains $\langle a+I\rangle$. Since $a \cdot R=0=R \cdot a,\langle a+I\rangle$ is clearly an ideal and $a+I \neq 0$. Let $J$ be a nonzero ideal of $R / I$. Then there exist $y \in A$ and an integer $n$ such that $0 \neq y+n x+I \in J$, and we can choose $n=1$ or $n=0$. If $n=1$ then $(y+x+I)(x+I)=a+I \in J$ as claimed. Suppose $n=0$. Then $y \in A \backslash C$ and $G=P+C$ with $P \leqslant A$ implies $y=z+i$ with $0 \neq z \in P$ and $i=y-z \in C \cap A=I$. Since $y \notin I$ we have $z \notin B$. From $(P / B)[2]=\langle a+B\rangle$ we infer $2^{m} z+B=a+B$ for some positive integer $m$. Thus

$$
a+I=2^{m} z+I=2^{m}(z+i)+I=2^{m} y+I \in J
$$

completing the proof.

\section{The Proof of 3.3}

Throughout, we let $T=\bigoplus_{n<\omega} Z\left(2^{n}\right)$, and $\widehat{T}$ denotes the 2 -adic completion of $T$. Then $T \leqslant \widehat{T} \leqslant \prod_{n<\omega} Z\left(2^{n}\right)$, and every endomorphism $\phi$ of $T$ can be extended uniquely to an endomorphism of $\widehat{T}$. Let $\pi: \widehat{T} \rightarrow \widehat{T} / T$ denote the natural epimorphism. Note that the group $\widehat{T} / T$ is divisible.

We start with a general construction using ideas from [2].

A. Let $B$ be any torsion-free group such that $E n d B=\mathbf{Z} \cdot 1_{B}$, and let $f: B \rightarrow \widehat{T}$ be a set function. If $\pi \circ f \in \operatorname{Hom}(B, \widehat{T} / T)$ is a homomorphism we say that $f$ is eligible. Assume $f$ is eligible. Define

$$
B_{f}=\{(t+f(b), b) \mid t \in T, b \in B\} \subseteq \widehat{T} \oplus B
$$

One verifies the following. We will identify $\widehat{T}$ with $\widehat{T} \oplus 0$. 
4.1. $B_{f}$ is a subgroup of $\widehat{T} \oplus B$ with torsion part $t B_{f}=T$, and $B_{f} / t B_{f} \simeq B$.

4.2. $E n d B_{f}=Z \cdot 1_{B_{f}} \oplus \operatorname{Hom}\left(B_{f}, T\right)$.

4.3. If $g: B \rightarrow \widehat{T}$ is a set function such that $\pi \circ g=\pi \circ f$ then $B_{g}=B_{f}$.

4.4. $B_{f}$ splits if and only if there exists a homomorphism $\eta: B \rightarrow \widehat{T}$ such that $\pi \circ f=\pi \circ \eta$.

For $\phi \in \operatorname{Bom}(T, T)$, its unique extension to $\widehat{T}$ will be denoted by $\widehat{\phi}$. Note that $f$ eligible implies $\hat{\phi} \circ f$ eligible.

4.5. Given $\phi \in \operatorname{Hom}(T, T)$, there exists $\theta \in \operatorname{Hom}\left(B_{f}, T\right)$ extending $\phi$ if and only if $B_{\widehat{\phi} \circ f}$ splits.

Proof: Suppose, firstly, that $B_{\hat{\phi} \circ f}$ splits. It follows that there exists a homomorphism $\sigma: B_{\widehat{\phi} \circ f} \rightarrow T$ such that $\sigma \circ \iota=1_{T}$ where $\iota: T \rightarrow B_{\widehat{\phi} \circ f}$ denotes the inclusion map. One verifies that $\hat{\phi} \oplus 1_{B} \in \operatorname{End}(\widehat{T} \oplus B)$ induces a homomorphism from $B_{f}$ to $B_{\widehat{\phi} \circ f}$. Let $\theta=\sigma \circ\left(\hat{\phi} \oplus 1_{B}\right) \mid B_{f}$. Conversely, suppose there exists a $\theta$ as stated. In particular, $\theta(t, 0)=\phi(t)$ for all $t \in T$. Define $\eta: B \rightarrow \widehat{T}$ by $\eta(b)=\widehat{\phi}(f(b))-\theta(f(b), b)$ for all $b \in B$. Then $\pi \circ \eta=\pi \circ \widehat{\phi} \circ f$. By (4), it suffices to show $\eta$ is a homomorphism. Let $b, b^{\prime} \in B$. There exists $t \in T$ such that $f\left(b+b^{\prime}\right)=f(b)+f\left(b^{\prime}\right)+t$. Hence

$$
\eta\left(b+b^{\prime}\right)=\widehat{\phi}\left(f(b)+f\left(b^{\prime}\right)+t\right)-\theta\left(f(b)+f\left(b^{\prime}\right)+t, b+b^{\prime}\right)=\eta(b)+\eta\left(b^{\prime}\right) .
$$

B. We now specify the torsion-free group $B$. Let $J_{2}$ denote the ring of 2-adic integers, and let $\rho \in J_{2}$ be transcendental over the rational integers $Q$. For each natural number $n$ we have

$$
\rho=s_{n}+2^{n} \rho_{n}
$$

with $s_{n} \in \mathbf{Z}$ and $\rho_{n} \in J_{2}$. Let

$$
B=\left\langle\{1\} \cup\left\{\frac{\rho-s_{n}}{2^{n}} \mid n<\omega\right\}\right\rangle,
$$

and let $R$ be a subring of $\mathbf{Z}_{(2)}$, the integers localised at 2 , containing $\mathbf{Z}$. The set $R B=\{r b \mid r \in R, b \in B\} \subseteq J_{2}$ is an additive group.

\section{6. $\operatorname{End}(R B)=R \cdot 1_{R B}$.}

Proof: Let $\varepsilon$ be an endomorphism of $R B$ and $\gamma=\varepsilon(1) \in R B$. Since, for each $n, \rho=s_{n}+2^{n} \rho_{n}, \varepsilon(\rho)=\varepsilon\left(s_{n}\right)+2^{n} \varepsilon\left(\rho_{n}\right)=s_{n} \varepsilon(1)+2^{n} \rho_{n}^{\prime}=s_{n} \gamma+2^{n} \rho_{n}^{\prime}$. Hence $\varepsilon(\rho)=$ $\lim _{n \rightarrow \infty} s_{n} \gamma=\rho \gamma$. It follows that $\varepsilon$ is the multiplication by $\gamma$. There exists a positive integer $k$ such that $2^{k} \gamma=r+t \rho$ with $r$ and $t$ in $R$. Then $\varepsilon\left(2^{k} \rho\right)=2^{k} \rho \gamma=r \rho+t \rho^{2}$. Since $\rho$ is transcendental over $Q$, we have $t=0$ and $\gamma \in \mathbf{R}$. 
Let $\Phi=E n d T \backslash H m_{b}(T, T)$ denote the set of all endomorphisms of $T$ with unbounded image. Then $\Phi$ has cardinality $2^{N_{0}}$ so that we can fix an enumeration

$$
\Phi=\left\{\phi_{\alpha} \mid \alpha<2^{\omega}\right\} .
$$

4.7. For each $\alpha<2^{\omega}$, there exists an eligible set function $f_{\alpha}: B \rightarrow \widehat{T}$ such that $\phi_{\alpha} \in \Phi$ cannot be extended to a homomorphism from $(B)_{f_{\alpha}}$ to $T$.

Proof: Let $\phi=\phi_{\alpha} \in \Phi$. First of all, note that there exists an element $x=x_{\alpha}$ in $\widehat{T}$ such that $\widehat{\phi}(x) \in \widehat{T} \backslash T$ : being an unbounded subgroup of $T$, the image of $\phi$ contains a subgroup $C=\bigoplus_{i<\omega}\left(\phi\left(t_{i}\right)\right\rangle$ with $2^{2 i} \phi\left(t_{i}\right) \neq 0$ and $o\left(\phi\left(t_{i}\right)\right)<o\left(\phi\left(t_{i+1}\right)\right)$ for all $i$; if $x=\lim _{n \rightarrow \infty}\left(2 t_{1}+\cdots+2^{n} t_{n}\right)$ then $\widehat{\phi}(x)$ has infinite order. Since $F=\langle 1, \rho\rangle$ is a free subgroup of $B$, there exists a homomorphism $\mu$ from $F$ to $\widehat{T}$ such that $\mu(1)=0$ and $\mu(\rho)=x$. Then $\pi \circ \mu: F \rightarrow \widehat{T} / T$ is a homomorphism which, since $\widehat{T} / T$ is divisible, can be extended to a homomorphism $\psi \in H o m(B, \widehat{T} / T)$. For each $b \in B \backslash F$ choose $t_{b} \in \widehat{T}$ such that $\psi(b)=\pi\left(t_{b}\right)$. The mapping $b \mapsto t_{b}$ extends $\mu$ to a function $f=f_{\alpha}: B \rightarrow \widehat{T}$ with $f \mid F=\mu$ which is eligible. Assume $\phi$ can be extended to a homomorphism from $B_{f}$ to $T$. By 4.4 and 4.5 , there exists $\eta \in H \circ m(B, \widehat{T})$ such that $\pi \circ \widehat{\phi} \circ f=\pi \circ \eta$. Hence, $\pi \eta(1)=\pi \widehat{\phi} f(1)=\pi \widehat{\phi}(0)=0$ which implies $\eta(1)=s \in T$; similarly, $\pi \eta(\rho)=\pi \widehat{\phi}(x)$ so that $\eta(\rho)=\widehat{\phi}(x)+t$ for some $t \in T$. As before, $\eta(\rho)=\eta\left(s_{n}+2^{n} \rho_{n}\right)=s_{n} \eta(1)+2^{n} \rho_{n}^{\prime}$ for all $n$. It follows that $\widehat{\phi}(x)+t=\lim _{n \rightarrow \infty} s_{n} s=s \rho \in T$ which contradicts $\widehat{\phi}(x) \notin T$.

C. Let $K_{2}$ denote the field of 2-adic numbers. Since $K_{2}$ has transcendence degree $2^{\mathfrak{K}_{0}}$ over the rationals, there exists a subset $I I \subseteq J_{2}$ of cardinality $2^{\mathfrak{K}_{0}}$ which is algebraically independent over $\mathbf{Z}$. Fix an enumeration

$$
I I=\left\{\pi_{\alpha} \mid \alpha<2^{\omega}\right\},
$$

and let, for each $\alpha$ and each natural number $n, \pi_{\alpha}=s_{n}^{\alpha}+2^{n} \rho_{n}^{\alpha}$ with $s_{n}^{\alpha} \in \mathbf{Z}$ and $\rho_{n}^{\alpha} \in J_{2}$. For each $\alpha<2^{\omega}$, let $B_{\alpha}$ be the group $B$ constructed in $B$ with $\rho=\pi_{\alpha}$. Well known set theoretical arguments show the existence of a family $\mathcal{F}$ of sets of rational primes with the following properties: (i) no set in $\mathcal{F}$ contains the prime 2 or the prime 3; (ii) no set in $\mathcal{F}$ is properly contained in another one; and (iii) $\mathcal{F}$ has cardinality $2^{K_{0}}[6]$. (The following argument shows that every countably infinite set $S$ has a family $\mathcal{F}$ of subsets satisfying (ii) and (iii): given $n \in N$, let $S_{n}$ denote the set of all functions $f:\{1, \ldots, n\} \rightarrow \mathrm{N}$. Then each $S_{n}$ is countable and so is their union $S=\bigcup_{n \in \mathbb{N}} S_{n}$. Let $T=\mathbf{N}^{\mathbf{N}}$ be the set of all functions from $\mathbf{N}$ to $\mathbf{N}$. Then $|T|=2^{\aleph_{0}}$. For $g \in T$, let $I(g)=\{f \in S|f|\{1, \ldots, n\}=g \mid\{1, \ldots, n\}$ for some $n \in \mathrm{N}\}$. Let $\mathcal{F}=\{I(g) \mid g \in T\}$.) Again, choose an indexing $\mathcal{F}=\left\{\Delta_{\alpha} \mid \alpha<2^{\omega}\right\}$. As customary, for $p$ a prime, $\mathbf{Q}^{(p)}$ denotes the set of all rational numbers with denominator a power of 
p. For each $\alpha<2^{\omega}$, let $\mathbf{Q}_{\alpha}=\sum\left\{\mathbf{Q}^{(p)} \mid p \in \Delta_{\alpha}\right\}$, a subring of $\mathbf{Q}$. Define a subgroup $H$ of the external direct sum $\bigoplus_{\alpha<2^{\omega}} K_{2}$ as follows: if $e_{\beta}$ denotes the vector with 1 in the beta-th coordinate and zeros elsewhere, we let

$$
H=\bigoplus_{\alpha<2^{\omega}} \mathbf{Q}_{\alpha} B_{\alpha} e_{\alpha}+\sum_{1<\alpha<2^{\omega}} \mathbf{Q}^{(2)}\left(e_{\alpha}-e_{0}\right)+\mathbf{Q}^{(3)}\left(e_{1}-e_{0}\right) .
$$

Let $\sigma=t\left(Q^{(2)}\right)$ denote the type of $Q^{(2)}$, let $\tau=t\left(Q^{(3)}\right)$ and let $\tau_{\alpha}=t\left(Q_{\alpha}\right)$. One verifies the following. For $t$ a type, $H(t)$ denotes the (fully invariant) subgroup of $H$ consisting of all elements of type greater than or equal to $t$. The pure subgroup generated by a subgroup $A$ is denoted by $A_{*}$.

4.8. The following hold:
(1) $\quad H(\sigma)=\left(\sum_{1<\alpha<2^{\omega}} Q^{(2)}\left(e_{\alpha}-e_{0}\right)\right)$.
(2) $\quad H(\tau)=\left(\mathrm{Q}^{(3)}\left(e_{1}-e_{0}\right)\right)$.
(3) For each $\alpha<2^{\omega}, H\left(\tau_{\alpha}\right)=Q_{\alpha} B_{\alpha} e_{\alpha}$.

Proof of (1): Put $A=\sum_{1<\alpha<2 \omega} \mathbf{Q}^{(2)}\left(e_{\alpha}-e_{0}\right)$. Then $A \leqslant H(\sigma)$. Let $w \in H$ be an element of infinite 2-height. Without loss of generality, we may assume that $w$ is an element of $\bigoplus_{\alpha<2^{\omega}} Q_{\alpha} B_{\alpha} e_{\alpha}+Q^{(s)}\left(e_{1}-e_{0}\right)$. In generalised vector notation,

$$
w=\left(r_{\alpha}+t_{\alpha} \rho_{n_{\alpha}}^{\alpha}+p_{\alpha}\right)
$$

with $r_{\alpha}, t_{\alpha} \in Q_{\alpha}, n_{\alpha}$ positive integers, $p_{\alpha} \in Q^{(3)}, p_{0}+p_{1}=0$ and $p_{\alpha}=0$ for all $\alpha>1$. In fact, we may assume that all of $r_{\alpha}, t_{\alpha}, p_{\alpha}$ are integers, and $p_{\alpha}=0$ for all $\alpha$. Also, since only finitely many components of $w$ are nonzero, it is possible to write $w$ such that $n_{\alpha}=n_{\beta}=n \in \mathbf{N}$ for all $\alpha$ and $\beta$. By hypothesis, given any $m \in \mathbf{N}$, there exists $y_{m} \in H$ such that $2^{m} y_{m}=w$, and

$$
y_{m}=\left(r_{\alpha}^{m}+t_{\alpha}^{m} \rho_{n_{m}}^{\alpha}+q_{\alpha}^{m}+p_{\alpha}^{m}\right)
$$

with $r_{\alpha}^{m}, t_{\alpha}^{m} \in \mathbf{Q}_{\alpha}, q_{\alpha}^{m} \in \mathbf{Q}^{(2)}, q_{1}^{m}=0, \sum_{\alpha} q_{\alpha}^{m}=0, p_{\alpha}^{m} \in \mathbf{Q}^{(3)}, p_{0}^{m}+p_{1}^{m}=0$ and $p_{\alpha}^{m}=0$ for all $\alpha>1$. Thus, for all $\alpha$ and all $m$,

$$
2^{m}\left(r_{\alpha}^{m}+t_{\alpha}^{m} \rho_{n_{m}}^{\alpha}+q_{\alpha}^{m}+p_{\alpha}^{m}\right)=r_{\alpha}+t_{\alpha} \rho_{n}^{\alpha}
$$

which implies

$$
2^{m+n+n_{m}}\left(r_{\alpha}^{m}+q_{\alpha}^{m}+p_{\alpha}^{m}\right)+2^{m+n} t_{\alpha}^{m}\left(\pi_{\alpha}-s_{n_{m}}^{\alpha}\right)=2^{n+n_{m}} r_{\alpha}+2^{n_{m}} t_{\alpha}\left(\pi_{\alpha}-s_{n}^{\alpha}\right) .
$$

The linear independence of the $\pi_{\alpha}$ over $Q$ implies, for all $\alpha$ and all $m$,

$$
2^{m+n} t_{\alpha}^{m}=2^{n_{m}} t_{\alpha}
$$


and, substituting,

$$
2^{m+n+n_{m}}\left(r_{\alpha}^{m}+q_{\alpha}^{m}+p_{\alpha}^{m}\right)-2^{n_{m}} t_{\alpha} s_{n_{m}}^{\alpha}=2^{n+n_{m}} r_{\alpha}-2^{n_{m}} t_{\alpha} s_{n}^{\alpha} .
$$

Assume, by way of contradiction, that $t_{\beta} \neq 0$ for some $\beta$. Then $t_{\beta}=\left(2^{k} p\right) / q$ for some integers $k \geqslant 0$ and $p$ and $q$ odd. Since $t_{\beta}^{m}$ has odd denominator, (i) implies $n_{m}+k \geqslant m+n$. Note that $n$ and $k$ are fixed. Thus

$$
\lim _{m \rightarrow \infty} n_{m}=\infty
$$

From (ii) we obtain

$$
2^{m+n} q_{\alpha}^{m}=2^{n} r_{\alpha}-t_{\alpha} s_{n}^{\alpha}+t_{\alpha} s_{n_{m}}^{\alpha}-2^{m+n}\left(r_{\alpha}^{m}+p_{\alpha}^{m}\right) .
$$

Since, for all $m$, the denominator of $r_{\alpha}^{m}+p_{\alpha}^{m}$ is odd, (iii) and (iv) imply

$$
\lim _{m \rightarrow \infty}\left(2^{n+m} q_{\alpha}^{m}\right)=2^{n} r_{\alpha}-t_{\alpha} s_{n}^{\alpha}+t_{\alpha} \pi_{\alpha} .
$$

For each $m$, let $s_{m}=\sum_{\alpha} 2^{n+m} q_{\alpha}^{m}$. Since $\sum_{\alpha} q_{\alpha}^{m}=0$, each $s_{m}$ is zero. Using $(v)$ one verifies

$$
0=\lim _{m \rightarrow \infty} s_{m}=\sum_{\alpha}\left(2^{n} r_{\alpha}-t_{\alpha} s_{n}^{\alpha}+t_{\alpha} \pi_{\alpha}\right),
$$

and the linear independence of the $\left\{\pi_{\alpha}\right\}$ over $Q$ implies $t_{\alpha}=0$ for each $\alpha$. Because of (i), all $t_{\alpha}^{m}$ are zero and from (iv) we infer $0=2^{m} \sum_{\alpha} q_{\alpha}^{m}=\sum_{\alpha} r_{\alpha}-2^{m} \sum_{\alpha}\left(r_{\alpha}^{m}+p_{\alpha}^{m}\right)$. It follows that $\sum_{\alpha} r_{\alpha}$ has infinite 2-height in the ring $\mathbf{Q}^{(3)}+\sum_{\alpha} \mathbf{Q}_{\alpha}$ which implies $\sum_{\alpha} r_{\alpha}=0$. Thus, $w=\sum_{\alpha}^{\alpha} r_{\alpha} e_{\alpha}=\sum_{\alpha} r_{\alpha}\left(e_{\alpha}-e_{0}\right)=z+k\left(e_{1}-e_{0}\right)$ with $z=\sum_{1<\alpha<2 \omega} r_{\alpha}\left(e_{\alpha}^{\alpha}-e_{0}\right) \epsilon$ $\sum_{1<\alpha<2 \omega} \mathrm{Z}\left(e_{\alpha}-e_{0}\right) \leqslant H(\sigma)$ and $k=r_{1} \in \mathbf{Z}$. Hence, for all $m, k / 2^{m} \in \mathbf{Q}_{1}+\mathbf{Q}^{(3)}$ which implies $k=0$. We have shown that $w \in A$.

Proof of (2): Obviously, $Q^{(3)}\left(e_{1}-e_{0}\right) \leqslant H(\tau)$. Let $w=\left(r_{\alpha}+t_{\alpha} \rho_{n}^{\alpha}+q_{\alpha}\right) \epsilon$ $H(\tau)$ with integers $r_{\alpha}, t_{\alpha}, q_{\alpha}$; we may assume each $q_{\alpha}$ is zero. We use the same notation as before: there exist $y_{m}=\left(r_{\alpha}^{m}+t_{\alpha}^{m} \rho_{n_{m}}^{\alpha}+q_{\alpha}^{m}+p_{\alpha}^{m}\right) \in H$ such that $3^{m} y_{m}=w$. Corresponding to (i) we obtain $3^{m} 2^{n} t_{\alpha}^{m}=2^{n_{m}} t_{\alpha}$ which shows that each $t_{\alpha}$ has infinite 3-height in $Q_{\alpha}+Q^{(2)}$. Hence $t_{\alpha}=0$ for each $\alpha$ and $w=\left(r_{\alpha}\right)$, $y_{m}=\left(r_{\alpha}^{m}+q_{\alpha}^{m}+p_{\alpha}^{m}\right)$. For $\alpha>1, p_{\alpha}^{m}=0$ which implies that $r_{\alpha}=3^{m}\left(r_{\alpha}^{m}+q_{\alpha}^{m}\right)$ has infinite 3 -height in $Q_{\alpha}+Q^{(2)}$. It follows that $r_{\alpha}=0$ for $\alpha>1$. Hence $r_{0}+r_{1}=\sum_{\alpha} r_{\alpha}=\sum_{\alpha} 3^{m}\left(r_{\alpha}^{m}+q_{\alpha}^{m}+p_{\alpha}^{m}\right)=3^{m}\left(\sum_{\alpha} r_{\alpha}^{m}+\sum_{\alpha} q_{\alpha}^{m}+\sum_{\alpha} p_{\alpha}^{m}\right)=3^{m} \sum_{\alpha} r_{\alpha}^{m}$ 
has infinite 3-height in $\sum_{\alpha} Q_{\alpha}$. Thus, $r_{0}+r_{1}=0$ and $w=r_{1}\left(e_{1}-e_{0}\right) \in Z\left(e_{1}-e_{0}\right)$ as desired.

Proof of (3): Fix $\beta<2^{\omega}$ and let $w \in H\left(\tau_{\beta}\right)$. Assume, by way of contradiction, there exists an $\alpha \neq \beta$ belonging to the support of $w$. By hypothesis, there exists a prime $p$ such that $p \in \Delta_{\beta}$ but $p \notin \Delta_{\alpha}$, and $p \neq 2,3$. For each positive integer $m$, there exists $y_{m} \in H$ such that $p^{m} y_{m}=w$. Using the same notation as before, letting $w=\left(r_{\alpha}+t_{\alpha} \rho_{n}^{\alpha}+q_{\alpha}+p_{\alpha}\right)$ and $y_{m}=\left(r_{\alpha}^{m}+t_{\alpha}^{m} \rho_{n_{m}}^{\alpha}+q_{\alpha}^{m}+p_{\alpha}^{m}\right)$, the equation corresponding to (i) is $p^{m} 2^{n} t_{\alpha}^{m}=2^{n_{m}} t_{\alpha}$ for all $m$ which shows that $t_{\alpha}=0=t_{\alpha}^{m}$, and $r_{\alpha}+q_{\alpha}+p_{\alpha}=p^{m}\left(r_{\alpha}^{m}+q_{\alpha}^{m}+p_{\alpha}^{m}\right)$ has infinite $p$-height in $\mathbf{Q}_{\alpha}+\mathbf{Q}^{(2)}+\mathbf{Q}^{(3)}$. Thus, the $\alpha$-th component of $w$ is zero and $w=\left(r_{\beta}^{m}+t_{\beta}^{m} \rho_{n}^{\beta}+q_{\beta}^{m}+p_{\beta}^{m}\right) e_{\beta}$. Since, for $\alpha \neq \beta$, $r_{\alpha}+q_{\alpha}+p_{\alpha}=0$, both $q_{\alpha}$ and $p_{\alpha}$ are integers. Hence, so are $q_{\beta}=-\sum_{\alpha \neq \beta} q_{\alpha}$ and $p_{\beta}=-\sum_{\alpha \neq \beta} p_{\alpha}$. It follows that $w \in Q_{\beta} B_{\beta} e_{\beta}$.

4.9. $E n d H=Z \cdot 1_{H}$.

Proof: Let $\varepsilon \in E n d H$. By 4.8(3), for each $\alpha, \varepsilon$ induces an endomorphism in $\mathbf{Q}_{\alpha} B_{\alpha} e_{\alpha}$ which, by 4.6 , must be the multiplication by some $r_{\alpha} \in \mathbf{Q}_{\alpha}$. Pick $\beta>1$. By 4.8(1), we have

$$
\varepsilon\left(e_{\beta}-e_{0}\right)=r_{\beta} e_{\beta}-r_{0} e_{0} \in\left(\sum_{1<\alpha<2^{\omega}} \mathbf{Q}^{(2)}\left(e_{\alpha}-e_{0}\right)\right) * .
$$

Thus, there exists a nonzero integer $n$ such that $n\left(r_{\beta} e_{\beta}-r_{0} e_{0}\right)=\sum_{1<\alpha} q_{\alpha}\left(e_{\alpha}-e_{0}\right)$. It follows that $r_{\beta}=n^{-1} q_{\beta}=r_{0}$. Similarly, using 4.8(2), $r_{1}=r_{0}$. It follows that $\varepsilon$ restricted to $\bigoplus_{\alpha<2^{\omega}} \mathbf{Q}_{\alpha} B_{\alpha} e_{\alpha}$ is the multiplication by $r_{0} \in \mathrm{Z}$. The latter subgroup being full in $B$ shows $\varepsilon=r_{0} \cdot 1_{H}$.

4.10. $H=\left\langle e_{0}\right\rangle+\left\langle e_{1}\right\rangle+2 H$, and $H / 2 H$ has rank two.

Proof: Let $R$ be a subring of $Q$ such that every element in $R$ has odd denominator. Then $R=Z+2 R$. Since $\rho_{n}^{\alpha}=2 \rho_{n+1}^{\alpha}$, it follows that $\mathbf{Q}^{(3)}\left(e_{1}-e_{0}\right) \subseteq$ $\left\langle e_{0}\right\rangle+\left\langle e_{1}\right\rangle+2 H$ and, for each $\alpha<2^{\omega}$,

$$
\mathbf{Q}_{\alpha} B_{\alpha} e_{\alpha}=\mathbf{Q}_{\alpha}\left\langle 1, \rho_{n}^{\alpha}\right\rangle e_{\alpha} \subseteq \mathbf{Q}_{\alpha} e_{\alpha}+2 H \subseteq\left\langle e_{\alpha}\right\rangle+2 H .
$$

If $\alpha>1, e_{\alpha}=e_{0}+\left(e_{\alpha}-e_{0}\right) \in\left\langle e_{0}\right\rangle+2 H$. Thus, $H=\left\langle e_{0}\right\rangle+\left\langle e_{1}\right\rangle+2 H$. In order to show $e_{0}$ and $e_{1}$ are linearly independent modulo $2 H$, let $a$ and $b$ be integers such that $a e_{0}+b e_{1} \in 2 H$. Using the same symbolism as above, $a e_{0}+b e_{1}=2\left(r_{\alpha}+t_{\alpha} \rho_{n}^{\alpha}+q_{\alpha}+p_{\alpha}\right)$ and as before we must have $t_{\alpha}=0$ for all $\alpha$ and $r_{\alpha}+q_{\alpha}=0$ if $\alpha>1$ so that $q_{\alpha}$ 
must be an integer. This implies $q_{0}=-\sum_{1<\alpha} q_{\alpha}$ is an integer and $2 q_{0}$ is even. Since $q_{1}=0,2 p_{1}=b-2 r_{1} \in \mathbf{Q}^{(3)} \cap \mathbf{Q}_{1}=\mathbf{Z}$ which implies $p_{1}=-p_{0}$ is an integer. Since $r_{0}=\left(a-2 q_{0}-2 p_{0}\right) / 2$ has odd denominator, $a$ must be even. Similarly, $b$ must be even.

4.11. For every $\phi \in \operatorname{Hom}(H, T), \operatorname{Im} \phi$ is bounded.

PROOF: There exists a positive integer $n$ such that $2^{n} \phi\left(e_{i}\right)=0$ for $i=1,2$. By 4.10, $\phi(H)=\left\langle\phi\left(e_{0}\right)\right\rangle+\left\langle\phi\left(e_{1}\right)\right\rangle+2 \phi(H)$ which implies $2^{n} \phi(H)=2^{n+1} \phi(H)=0$ since $T$ is reduced.

D. We are getting ready to construct our group $G$. By 4.7, for each $\alpha<2^{\omega}$, there exists an eligible map $f_{\alpha}: B_{\alpha} \rightarrow \widehat{T}$ such that $\phi_{\alpha} \in \Phi$ does not extend to a homomorphism from $\left(B_{\alpha}\right)_{f_{\alpha}}$ to $T$. It follows that $\oplus f_{\alpha}: \bigoplus_{\alpha<2^{\omega}} B_{\alpha} \rightarrow \widehat{T}$ is an eligible map which, in turn, extends to an eligible map $f: H \rightarrow \widehat{T}$ since $\widehat{T} / T$ is divisible, hence injective. By 4.9, $H$ satisfies the same hypotheses as the group $B$ in part $\mathbf{A}$. Let $K=H_{f}$. Then

\subsection{2. $\operatorname{EndK}=\mathrm{Z} \cdot 1_{K} \oplus \operatorname{Hom}_{b}(K, T)$.}

ProOF: By 4.2, it suffices to show every homomorphism from $K$ to $T$ has bounded image. Let $\phi \in \operatorname{Hom}(K, T)$. By $4.1, t K=T$; let $\psi=\phi \mid t K$ be the restriction map and assume, by way of contradiction, that $\psi$ has unbounded image. Then $\psi=\phi_{\alpha}$ for some $\alpha<2 \omega$. By construction, $\phi_{\alpha}$ cannot be extended to $\left(B_{\alpha}\right)_{f_{\alpha}}$. Since $T \leqslant\left(B_{\alpha}\right)_{f_{\alpha}}$ and

$$
\left(B_{\alpha}\right)_{f_{\alpha}}=\left\{(t+f(b), b) \mid t \in T, b \in B_{\alpha}\right\} \leqslant H_{f}=K,
$$

$\phi_{\alpha}$ does not extend to $K$ which is a contradiction. Hence $2^{n} \phi(T)=0$ and $2^{n} \phi$ induces a homomorphism $\eta: K / T \rightarrow T$ given by $\eta(x+T)=2^{n} x$. It follows from 4.1 and 4.11 that $\eta$ is bounded and, hence, so is $\phi$.

Let $P$ be the Prüfer 2-group with $2^{\omega} P=\langle a\rangle$ as above. Then there is an exact sequence $0 \rightarrow\langle a\rangle \rightarrow P \rightarrow T \rightarrow 0$ the epimorphism of which induces an epimorphism $\operatorname{Ext}(H, P) \rightarrow \operatorname{Ext}(H, T)$. Thus, there exists a group $G$ and homomorphisms such that the following diagram is commutative with exact rows:

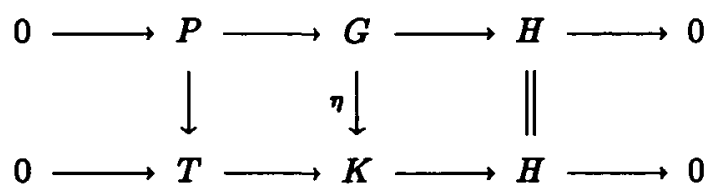

By the Five Lemma, $\eta$ is an epimorphism, and $\langle a\rangle$ is the kernel of $\eta$. Hence $K \simeq G /\langle a\rangle$. One verifies that $G /(t G+2 G) \simeq H / 2 H$ which, by 4.10 , has rank two. The group $G$ will satisfy the hypothesis of 3.2 if it has the property that $E n d G=\mathbf{Z} \cdot 1_{G} \oplus \operatorname{Hom}_{b}(G, P)$. 
Let $\varepsilon$ be an endomorphism of $G$. Since $\langle a\rangle=2^{\omega} t G$ is fully invariant in $G, \varepsilon$ induces an endomorphism $\bar{\varepsilon}$ in $G /\langle a\rangle \simeq K$. By 4.12, there exist integers $m$ and $n$ such that $2^{m} \bar{\varepsilon}=2^{m} n \cdot 1_{G /\langle a}$ and $m$ is positive. Hence $2^{m+1}\left(\varepsilon-n \cdot 1_{G}\right)=0$ as desired.

REMARK. A more elaborate construction along the same lines yields a group $G$ with $G /(t G+2 G)$ of dimension $2^{\mathrm{K}_{0}}$.

\section{REFERENCES}

[1] G. Birkenmeier and H. Heatherly, 'Rings whose additive endomorphisms are ring endomorphisms', Bull. Austral. Math. Soc. 42 (1990), 145-152.

[2] M. Dugas, P. Hill, and K.M. Rangaswamy, 'Butler groups of infinite rank II', Trans. Amer. Math. Soc. 320 (1990), 643-664.

[3] S. Feigelstock, 'Rings whose additive endomorphisms are multiplicative', Period. Math. Hungar. 19 (1988), 257-260.

[4] L. Fuchs, Infinite abelian groups, I (Academic Press,, New York, 1970).

[5] L. Fuchs, Infinite abelian groups, II (Academic Press, New York, 1973).

[6] T. Jech, Set theory (Academic Press, New York, 1978).

[7] K.H. Kim and F.W. Roush, 'Additive endomorphisms of rings', Period. Math. Hungar. 12 (1981), 241-242.

[8] N.H. McCoy, Rings and ideals, Carus Mathematical Monographs (The Mathematical Association of America, Fifth Impression, 1971.).

[9] R.P. Sullivan, 'Research problem No. 23', Period. Math. Hungar. 8 (1977), 313-314.

Baylor University

Waco TX 76798-7328

United States of America

University of Houston

Houston TX 77204-3476

United States of America
University of Houston

Houston TX 77204-3476

United States of America 